\title{
The multi-faceted nature of visual statistical learning: Individual differences in learning conditional and distributional regularities across time and space
}

\author{
Bethany Growns ${ }^{1,2} \cdot$ Noam Siegelman ${ }^{3} \cdot$ Kristy A. Martire ${ }^{2}$ \\ Published online: 23 July 2020 \\ (C) The Psychonomic Society, Inc. 2020
}

\begin{abstract}
Emerging research has demonstrated that statistical learning is a modality-specific ability governed by domain-general principles. Yet limited research has investigated different forms of statistical learning within modality. This paper explores whether there is one unified statistical learning mechanism within the visual modality, or separate task-specific abilities. To do so, we examined individual differences in spatial and nonspatial conditional and distributional statistical learning. Participants completed four visual statistical learning tasks: conditional spatial, conditional nonspatial, distributional spatial, and distributional nonspatial. Performance on all four tasks significantly correlated with each other, and performance on all tasks accounted for a large portion of the variance across tasks (57\%). Interestingly, a portion of the variance of task performance (between $11 \%$ and $18 \%$ ) was also accounted for by performance on each of the individual tasks. Our results suggest that visual statistical learning is the result of the interplay between a unified mechanism for extracting conditional and distributional statistical regularities across time and space, and an individual's ability to extract specific types of regularities.
\end{abstract}

Keywords Statistical learning $\cdot$ Domain-general mechanisms $\cdot$ Modality specificity $\cdot$ Individual differences $\cdot$ Psychometrics

Humans can extract and encode statistical regularities from perceptual input across visual, auditory, and even tactile modalities - after only brief exposure and without instruction to do so (Conway \& Christiansen, 2005; Fiser \& Aslin, 2001; Frost, Armstrong, \& Christiansen, 2019; Pavlidou \& Bogaerts, 2019). This is known as statistical learning and is broadly thought to underlie many basic perceptual and cognitive processes such as categorization and language acquisition (Siegelman \& Frost, 2015). Individuals are able to extract both conditional relationships between stimuli (e.g., A co-occurs

This work was supported by an Australian Government Research Training Program (RTP) Scholarship to Bethany Growns and an Australian Research Council Linkage Project Grant (LP160100008) to Kristy A. Martire.

Bethany Growns

bethany.growns@gmail.com

1 School of Social and Behavioural Sciences, Arizona State University, Phoenix, AZ, USA

2 University of New South Wales, Sydney, NSW, Australia

3 Haskins Laboratories, New Haven, CT, USA with B in time or space; i.e., conditional statistical learning) and distributions of stimuli (e.g., C is more frequent than D; i.e., distributional statistical learning; Siegelman, Bogaerts, Christiansen, \& Frost, 2017a; Thiessen \& Erickson, 2013). Individuals that are exposed to conditional or distributional statistical information typically perform above chance when deciding which stimuli is more familiar to them (e.g., AB. vs. XY, or C. vs. D vs. Z; Fiser \& Aslin, 2001; Growns \& Martire, 2020). These two forms of statistical learning are thought to be facilitated by different, but interrelated, memory processes (Thiessen \& Erickson, 2013; Thiessen, Kronstein, \& Hufnagle, 2013).

Statistical learning was initially conceptualized as a unified domain-general ability where learning is interdependent across all sensory modalities (see Frost, Armstrong, Siegelman, \& Christiansen, 2015 for review). However, emerging research suggests that statistical learning has both modality and stimuli-specific constraints (Conway \& Christiansen, 2005, 2006; Mitchel \& Weiss, 2011; Perfors \& Kidd, 2018; Raviv \& Arnon, 2018). For example, you may expect that statistical learning performance would change at the same rate across development in all modalities-yet visual statistical 
learning improves with age in children, whilst auditory statistical learning does not (Raviv \& Arnon, 2018). Modality-specific statistical learning is also supported by the fact that learning in one modality (e.g., visual) is not reliably associated with learning in other modalities (e.g., auditory) - as might be expected if statistical learning were one unified ability (Siegelman \& Frost, 2015).

Together, these converging lines of research demonstrate that statistical learning is not a unified, domain-general mechanism. Rather, they suggest that although statistical learning may be governed by domain-general principles, it is also subject to modality-specific constraints governed by respective brain regions (e.g., visual statistical learning would be governed by the visual cortex; Frost et al., 2015). Yet despite the breadth of research examining statistical learning across modalities, limited research has investigated it within modality.

Investigating statistical learning within modality is critical to furthering our understanding of it as a theoretical construct. Are different forms of statistical learning (i.e., conditional and distributional learning) part of one, unified ability within a modality? Or are they separate subprocesses affected by task-specific demands? If these forms of statistical learning are part of one modality-specific unified ability, individuals with high conditional learning might also have high distributional learning. Conversely, you would expect no relationship if they are separate subprocesses. In the same vein, although it is well documented that visual statistical learning can be demonstrated in both spatial and nonspatial tasks (Fiser \& Aslin, 2001, 2002), it is unclear whether the two types of learning are governed by the same mechanism. Given that the visual cortex shows enhanced sensitivity to spatial information (Chen \& Vroomen, 2013; Frost et al., 2015; Recanzone, 2009), individuals' performance in spatial tasks may be reliably associated with each other if spatial learning is also governed by a unified modality-specific mechanism (but not in nonspatial tasks).

In this paper, we examine whether spatial and nonspatial conditional and distributional statistical learning are governed by one unified visual statistical learning mechanism, or separate subprocesses within the visual modality. We examine individual differences in statistical learning to empirically explore the overlap or independence of these subprocesses. As discussed above, we would expect significant correlations between conditional and distributional learning in spatial and nonspatial tasks if statistical learning is one unified mechanism. Given the sensitivity of the visual cortex to spatial information, you may also predict stronger correlations between some tasks (i.e., spatial conditional and distributional learning) than others (e.g., spatial and nonspatial conditional learning). Conversely, we would expect no significant associations if visual statistical learning is comprised of several distinct subprocesses.

\section{Method}

\section{Design}

A 2 (conditional or distributional) $\times 2$ (nonspatial or spatial) within-subjects design was used. Participants completed the four tasks in the following set order to minimize error variance across participants (Mollon, Bosten, Peterzell, \& Webster, 2017): distributional nonspatial, conditional spatial, conditional nonspatial, distributional spatial. This experiment was preregistered, and the preregistration, data, and materials are available (https://osf.io/kua32/).

\section{Participants}

Participants were 76 undergraduate students from a large university in eastern Australia who were 19.30 years of age on average $(S D=2.14$ years, $\min =17, \max =28)$, and the majority of the sample was female $(65.8 \%)$. Participants received course credit for their participation and had normal or corrected-to-normal vision. We preregistered our intention to collect data from 67 participants, but recruited nine additional participants to replace data lost from the conditional spatial task due to a technical error.

\section{Tasks and dependent measures}

Each task consisted of an exposure and a test phase. During the exposure phase, participants viewed stimuli with predetermined statistical regularities. They were instructed to pay attention to the stimuli because they would be asked questions about them later. During each exposure phase, participants did not perform a cover task and were not informed about the presence of any statistical regularities.

During each test phase, participants completed pattern recognition and/or pattern completion trials to investigate how well they learned the predetermined regularities. During pattern recognition trials, participants were asked to choose which shape or shape-pattern was more familiar to them from arrays of two to four shapes/shape-patterns. During pattern completion trials, participants were asked to choose the shape that "best completes the pair" for a pattern with one missing target shape. Group-level chance performance was calculated by aggregating the different probabilities of responses for each trial.

Each task was replicated from validated tasks available in the literature (with minor modifications) wherever possible. Whilst this resulted in some differences in experimental parameters (e.g., trial numbers), we elected to use tasks that had previously demonstrated reliable assessment of statistical learning. Although we replicated experimental parameters and statistical regularities from the literature, each task in this paper used different shapes (see Fig. 1). This preserved the 


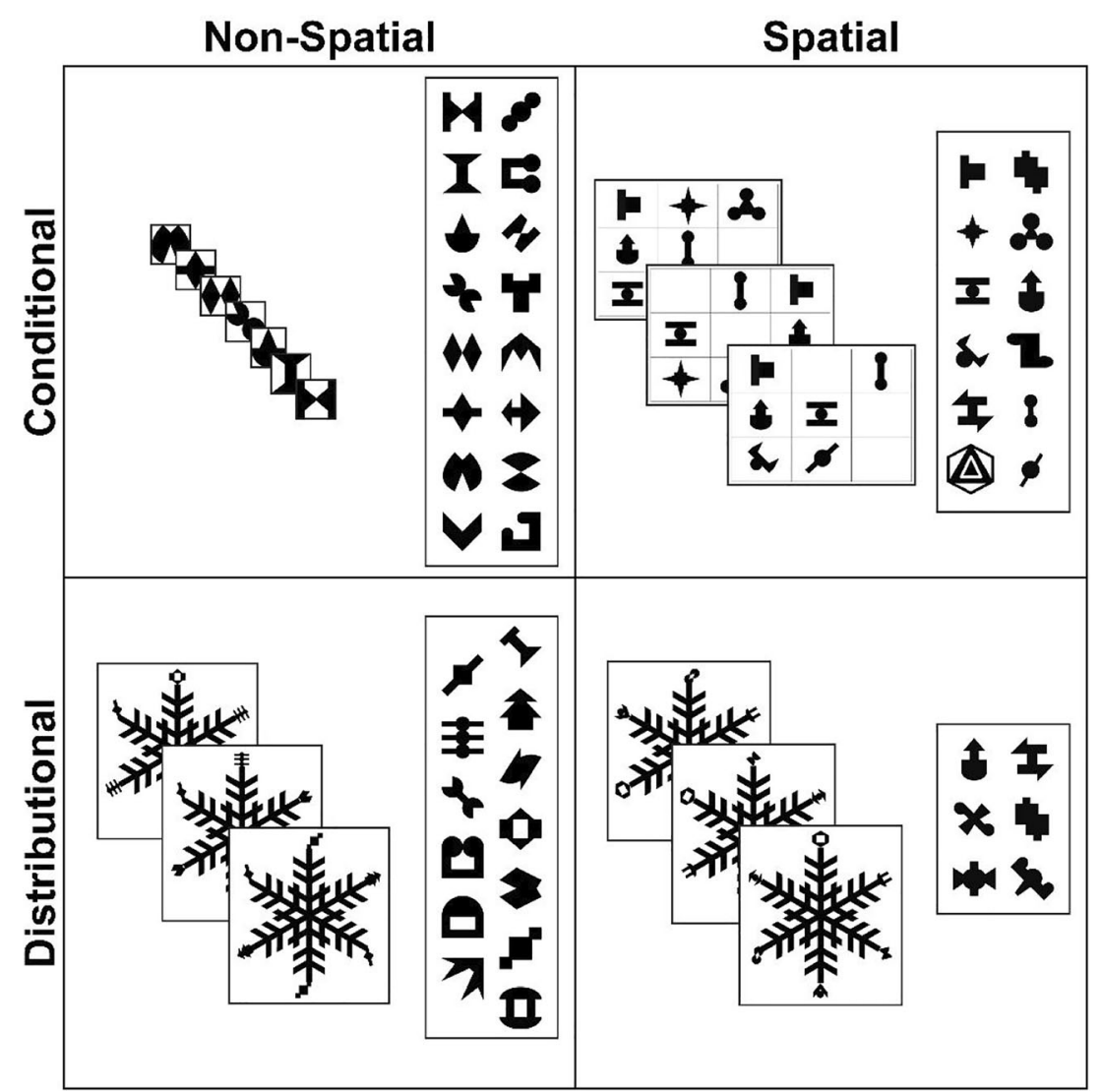

Fig. 1 Shapes and example stimuli used in the conditional nonspatial (upper left panel), conditional spatial (upper right panel), distributional nonspatial (lower left panel), and distributional spatial (lower right panel) tasks

independence of each task by ensuring there would be no interference across tasks.

Conditional nonspatial task Participants completed a conditional nonspatial task (see upper left panel of Fig. 1) replicated from Siegelman, Bogaerts, and Frost (2017b). This task has reliability and internal consistency within psychometrically recommended ranges (Cronbach's $\alpha=.88$, split-half reliability $=.83$ and test-retest reliability $=.68$; Siegelman, Bogaerts, et al., 2017).

Exposure. Participants completed one block where they viewed "triplets" of 16 individual shapes (see upper left panel of Fig. 1) in a temporal stream in a 10-minute exposure phase. Each shape was presented for $800 \mathrm{~ms}$, with an interstimulus interval (ISI) of $200 \mathrm{~ms}$. Conditional statistical information was manipulated so that shapes appeared together in one of eight predetermined triplets: four triplets made up of four shapes had transitional probabilities (TP) of .33 (i.e., 1-2-3, 2-1-4, 4-3-1, and 3-4-2); and four triplets made up of 12 shapes with TP of 1.0 (e.g., 5-6-7). Participants viewed each triplet twenty-four times in a randomized order, with the constraint that the same triplet could not be immediately repeated.
Test. Participants first completed 34 pattern recognition trials and then eight pattern completion trials (42 trials in total). On pattern recognition trials, participants were asked to choose the most familiar pair or triplet out of two or four pairs/triplets. On pattern completion trials, participants were asked to choose the shape "that best completes the triplet" from an array of three shapes. Participants were presented with pairs or triplets from exposure (with TPs of .33 or 1) and foils (i.e., incorrect answers with TPs from $0-.50$ ) with varying levels of position violations. They completed trials by pressing computer keys to select the most familiar pair/triplet or select the missing shape. Pattern recognition and completion trials were randomized within each block. Chance performance in this task was 16.67 trials, or $39.7 \%$ accuracy.

Conditional spatial task Participants completed a conditional spatial task (see upper right panel of Fig. 1) adapted from Experiment 2 in Fiser and Aslin (2001).

Exposure. Participants completed one block where they viewed shape-pairs made of 12 individual shapes in one 
hundred and forty-four $3 \times 3$ grids (see upper left panel of Fig. 1) presented in a randomized order in a 7-minute exposure phase. Grids were presented for 2,000 ms with no ISI. Conditional-spatial statistical information was manipulated so that shapes appeared together in one of six pairs of predetermined spatial relationships with spatial probabilities (SP) of 1. For example, Shapes 1 and 2 always co-occurred vertically (1 above 2 ), 3-4 always cooccurred horizontally (3 next to 4), and 5-6 always cooccurred obliquely ( 5 diagonal to 6 ). Six shape pairs cooccurred across all trials (two vertical, two horizontal, and two oblique). Each grid contained six shapes (made up from three pairs) by positioning pairs so that each pair neighboured at least one other. This ensured that conditional spatial learning was not facilitated by discernible segmentation cues. The shape-pairs always co-occurred in the same spatial relationship to ensure participants could extract pairs only based on spatial co-occurrences. Test. Participants completed 36 pattern recognition trials in a randomized order where they viewed two shape-pairs presented sequentially on each trial. ${ }^{1}$ They were asked to choose which shape pair was more familiar between exposure shape pairs (SPs of 1) and foil shape-pairs (i.e., two shapes that did not co-occur in exposure; with an SP of 0 ). Foils were two shapes that appeared in the tested positions of the grid during exposure but that never cooccurred in this spatial arrangement (i.e., $\mathrm{SP}=0$ ). Participants selected the most familiar shape-pair by pressing a computer key that corresponded to their choice. The correct answers were based on the SPs from the exposure phase. Chance performance in this task was 18 trials, or $50 \%$ accuracy.

Distributional nonspatial task Participants completed a distributional nonspatial task (see lower left panel of Fig. 1) replicated fromExperiment 1 in Growns \& Martire (in press). This task has reliability and internal consistency within psychometrically recommended ranges (Cronbach's $\alpha=.86$ and splithalf reliability $=.76$ derived from reanalysis of Growns \& Martire, in press; data available at https://osf.io/kua32/).

Exposure. Participants completed three blocks where they viewed 13 individual shapes that occurred on the "arms" of 60 complex patterns (see lower left panel in Fig. 1) in a 9-minute exposure phase. Each block was separated by a short self-determined break. Patterns were presented for $3000 \mathrm{~ms}$ (with a $1000 \mathrm{~ms}$ ISI) in a pseudorandomized order where all participants

\footnotetext{
${ }^{1}$ Note that participants did not complete pattern completion trials in the conditional spatial task as none were included in the original Fiser and Aslin (2001) task.
}

completed trials in one order randomly generated when coding the experiment. Distributional statistical information was manipulated so that each shape appeared with a different frequency across all the patterns that participants viewed. For example, Shape 1 appeared in all exemplars, 2 appeared in 0.3 of exemplars, 3 appeared in 0.7 of exemplars, and 4-13 each appeared in 0.1 of exemplars (see Fig. 2 for an example). These frequencies resulted in three distributional joint probability shape-pairs: $0.1(0.1$ $\times 1) ; 0.3(0.3 \times 1)$; and $0.7(0.7 \times 1)$. The entire set of 60 exemplars contained every possible spatial combination of shapes to ensure the distribution was independent of any spatial co-occurrences. As a consequence of every spatial combination being created whilst maintaining the distribution, Shapes 2 and 3 never co-occurred in the same exemplar (see Fig. 2).

Test. Participants completed 33 pattern recognition trials and 12 nonsequential pattern completion trials in a pseudorandomized order (45 trials in total). On pattern recognition trials, participants chose the most familiar shape or shape-pair out of two, three, or four shape/ shape-pairs. For 12 pattern recognition trials, participants chose between two shapes presented one after the other. For 21 pattern recognition trials, participants chose between two, three, or four shape-pairs presented simultaneously on-screen. On pattern completion trials, participants were asked to choose the shape "that best completes the pair" from an array of two, three, or four shapes. The shapes participants were presented on all trials occurred between 0.1 and 1 in the exposure phase, and the shape-pairs they chose between occurred between 0.1 and 0.7 . The correct answer was based on the frequency or joint probabilities from the exposure phase. They completed trials by pressing computer keys to either select the most familiar shape/shape-pair or select the missing shape. Participants were instructed to ignore shape orientation and location when making their choices. Chance performance in this task was 20.5 trials, or $45.5 \%$ accuracy.

Distributional spatial task Participants completed a distributional spatial task (see lower right panel of Fig. 1) that was created for the purposes of this study, but was adapted from the distributional nonspatial task in the literature (Growns \& Martire, in press).

Exposure. Participants completed three blocks where they viewed six individual shapes that occurred on the "arms" of 60 complex patterns (see lower right panel in 


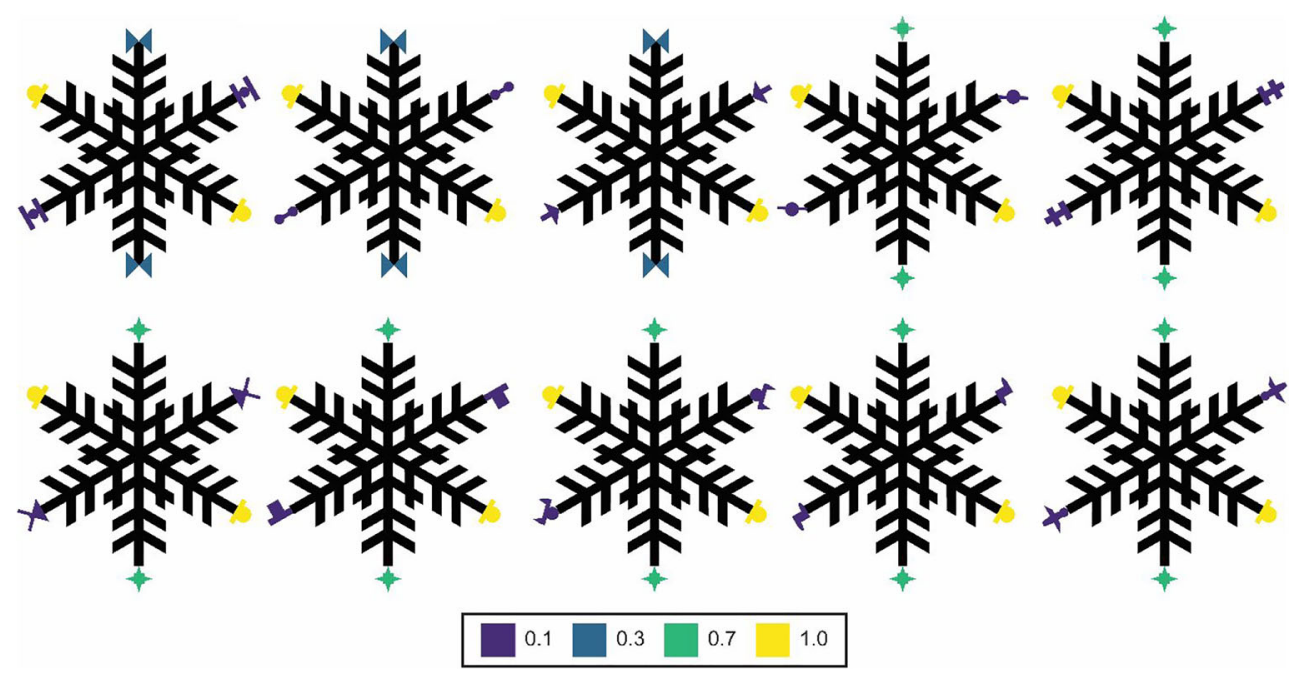

Fig. 2 Example of the distributional statistical information manipulated in patterns in the distributional nonspatial task. Note that exemplars in the task were presented in black and white

Fig. 1) presented in a pseudorandomized order in a 9minute exposure phase. Each block was separated by a short self-determined break and patterns were presented for 3,000 ms with a 1,000 ms ISI. Distributional-spatial statistical information was manipulated so that each shape appeared with different frequencies in different spatial locations across all patterns. Shapes occurred in these spatial locations: 1 appeared 0.1 on Arm $1^{2}$ and 0.9 on Arm 5; 2 appeared .02 on Arm 4 on 0.8 on Arm 2; 3 appeared 0.3 on Arm 3 and 0.7 on Arm 6; 4 appeared 0.1 on Arm 5, 0.4 on Arm 1, and 0.5 on Arm 3; 5 appeared 0.1 on Arms 2 and 6, 0.2 on Arm 1, and 0.6 on Arm 4; and 6 appeared 0.1 on Arm 2, 0.2 on Arms 3, 4, and 6, and 0.3 on Arm 1. The pseudorandom order of exemplars again ensured the spatial-frequency distribution was independent of any consistent temporal information.

Test. Participants completed 26 pattern recognition trials and 19 pattern completion trials in a pseudorandomized order (45 trials in total). On pattern recognition trials, participants chose the most familiar shape in a specific location out of an array of two, three, or four of the same shape in different spatial locations (see left panel of Fig. 3 ). Targets appeared most frequently in one spatial location (e.g., 0.9 on Arm 1), while foils were the same shape that appeared less frequently in another spatial location (e.g., 0.1 on Arm 5). On pattern completion trials, participants were asked to "choose the shape in the specific location that best completes the pair" from an array of two, three, or four of the same shape in different location (see right panel of Fig. 3). On all trials, the correct answer could only be determined by the location in which that

\footnotetext{
${ }^{2}$ Note "Arms" are labeled 1-6 in a clockwise order from the upper-left arm (Arm 1; see Fig. 1), upper-middle arm (Arm 2), upper-right arm (Arm 3), lower-right arm (Arm 4), lower-middle arm (Arm 5), and lower-left arm (Arm 6).
}

shape appeared most frequently. Chance performance in this task was 16.62 trials, or $36.9 \%$ accuracy.

\section{Procedure}

Participants provided demographic information and then completed each task in the set order (distributional nonspatial, conditional spatial, conditional nonspatial, and then distributional spatial). Each task consisted of an exposure and test phase as described above. Before each task, participants were instructed to pay attention to the stimuli as they would be asked some questions afterwards. Upon completion of the experiment, participants were thanked for their participation and debriefed.

\section{Results}

\section{Preregistered results}

Group-level analysis Statistical learning accuracy was significantly better than chance in all tasks (see Table 1). This indicates that participants successfully extracted and encoded the relevant statistical regularities in each task.

Individual-level analysis All tasks had internal consistency that was close to or within the recommended range for standard tests $(\alpha \mathrm{s}=0.76-0.92$; see Table 1$)$. This suggests that the measures generally produced reliable, internally consistent data and supports their use for assessment of individual differences.

All tasks also significantly correlated with one another (see Table 2 and Fig. 4). Importantly, the magnitude of the correlations did not significantly vary as a function of the tasks used despite the multiple comparisons in this analysis. For 


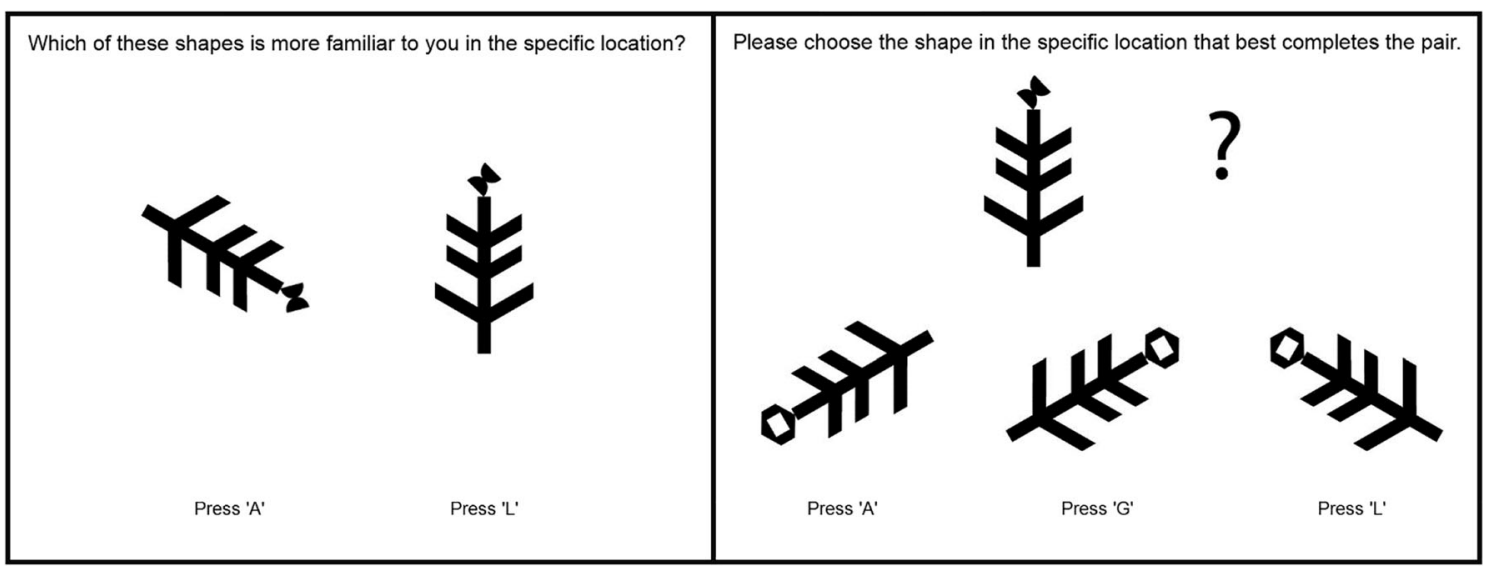

Fig. 3 Examples of pattern recognition (left panel) and completion (right panel) discrimination judgement trials

example, even the smallest $(r=.277)$ and largest $(r=.512)$ correlations did not significantly differ from one another (Fisher $r$-to- $z$ test: $Z=1.66, p=.097$ ). This suggests that all the tasks in this study share some portion of the variance in task performance and this shared variance does not vary significantly across all four tasks. However, it is also important to note that the correlations were not perfect, and were lower than the reliability coefficients for the same tasks (which present an upper-bound to the observed correlations). This suggests that each statistical learning task also taps into some portion of unique variance.

Given that we had no a priori predictions about the presence of any significant task correlations (see preregistration), we also calculated Bayes factors to examine the likelihood of the data under the null hypothesis (i.e., the absence of correlations) compared with an alternative hypothesis (i.e., the presence of correlations). We observed Bayes factors greater than 10 for 5 of the 6 correlations (see Table 2). This provides strong evidence for the presence of the correlations (Wetzels et al., 2011), although the remaining correlation (distribution$\mathrm{al} /$ conditional learning in nonspatial tasks) provided weaker support for the presence of the correlation.

\section{Exploratory results: Principle component analysis (PCA)}

We further explored the shared and unshared variance across all four tasks with a principle component analysis (PCA) using the prcomp function from the core stats package in $\mathrm{R}$. The loadings of all four tasks on the four components and the proportion of variance explained by each component can be seen in Table 3. The first factor explains the majority of variance in task performance observed across all four tasks $(57 \%)$. Note that all tasks are similarly loaded on this component, suggesting that it represents the shared component of figure variance across them.

Importantly, however, the next three components also explain substantial amount of observed variance $(43 \%$ overall; between $11 \%$ to $18 \%$ each). The task loadings suggest that these additional components reflect domain-specific variance, related specifically to the different tasks. The second component is strongly related to performance in the conditional spatial tasks; the third component is strongly related to performance in the distributional nonspatial tasks; and the fourth component differentiates performance in the conditional nonspatial and distributional spatial tasks (performance is positively related in the conditional nonspatial task and negatively related in the distributional spatial task). Overall, these results suggested that performance in each statistical learning task reflects a mixture of shared and nonshared variance.

\section{Discussion}

We examined whether spatial and nonspatial conditional and distributional statistical learning were part of one unified

Table 1. Descriptive statistics for statistical judgements in all tasks

\begin{tabular}{lllllll}
\hline & Chance & Mean $(S D)$ & $d f$ & $t$ & $p$ & Cronbach's $\alpha$ \\
\hline Conditional nonspatial & 16.67 & $22.39(6.98)$ & 75 & 7.15 & $<.001$ & .80 \\
Conditional spatial & 18 & $21.25(5.80)$ & 66 & 4.59 & $<.001$ & .76 \\
Distributional nonspatial & 20.50 & $34.64(8.43)$ & 75 & 14.62 & $<.001$ & .91 \\
Distributional spatial & 16.62 & $29.07(10.16)$ & 75 & 10.68 & $<.001$ & .92 \\
\hline
\end{tabular}


Table 2. Correlations between discrimination judgements in all tasks (Pearson correlations reported with p-values in brackets, with Bayes Factors displayed below)

\begin{tabular}{|c|c|c|c|c|}
\hline & Conditional nonspatial & Conditional spatial & Distributional nonspatial & Distributional spatial \\
\hline Conditional nonspatial & - & & & \\
\hline Conditional spatial & $\begin{array}{l}.475(<.001) \\
\mathrm{BF}=492.1\end{array}$ & - & & \\
\hline Distributional nonspatial & $\begin{array}{l}.277(.015) \\
\mathrm{BF}=2.55\end{array}$ & $\begin{array}{l}.365(.002) \\
\mathrm{BF}=13.9\end{array}$ & - & \\
\hline Distributional spatial & $\begin{array}{l}.512(<.001) \\
\mathrm{BF}>1,000\end{array}$ & $\begin{array}{l}.468(<.001) \\
\mathrm{BF}=370.4\end{array}$ & $\begin{array}{l}.335(.003) \\
\mathrm{BF}=10.5\end{array}$ & - \\
\hline
\end{tabular}

visual statistical learning mechanism, or separate subprocesses within the visual modality. Validating our tasks for the assessment of individual differences, we identified better-than-chance and generally reliable $(\alpha \mathrm{s}=.76-92)$ performance in all four tasks, including the novel distributional spatial task. Performance on all four tasks significantly correlated with one another, and a large portion of the variance across all four tasks was accounted for by one factor that all tasks similarly loaded on to. This demonstrates that on one level, at least some of the ability to learn spatial and nonspatial conditional and distributional regularities can be traced back to one unified visual statistical learning ability.

Importantly, however, our results also provide evidence that individual ability in separate tasks accounts for a substantial amount of variance. This is reflected by the moderate correlations observed between all tasks, as well as in the results of the PCA where performance on the conditional spatial and distributional nonspatial tasks discriminates between performance on all other tasks on two factors; and the remaining factor discriminates performance between the conditional nonspatial and distributional spatial tasks. Overall, our results suggest visual statistical learning is an interplay between a unified ability to learn spatial and nonspatial conditional and distributional regularities, and individual skill in extracting specific regularities.

Our findings thus extend the conceptualization of statistical learning as a modality-constrained ability governed by domain-general principles (Frost et al., 2015). They provide evidence that even within the visual modality, statistical learning performance can be traced back to a single unified ability, as well as to specific regularity computations. It also supports theoretical conceptualization of conditional and distributional statistical learning being underpinned by interrelated-yet separate-memory processes (Thiessen \& Erickson, 2013; Thiessen et al., 2013). We also identified a significant relationship between performance on both spatial and nonspatial tasks. The visual cortex is more sensitive to spatial regularities compared with nonspatial information-such as the conditional nonspatial task (Chen \& Vroomen, 2013; Frost et al., 2015; Recanzone, 2009). Our results demonstrate that the ability to learn spatial and nonspatial regularities are partially related to a single general visual statistical learning ability.

To our knowledge, this is the first empirical evidence that visual statistical learning is both a single unified ability to learn different forms of statistical regularities and individual ability to extract specific types of statistical regularities. It extends research on modality-specific constraints of statistical learning (Frost et al., 2015; Siegelman \& Frost, 2015) and work on differences between learning adjacent and nonadjacent auditory regularities that document the interplay between modality-specific, domain-specific, and general statistical learning computations (Newport \& Aslin, 2004; Romberg \& Saffran, 2013; Siegelman \& Frost, 2015).

\section{Limitations and future directions}

It is important to note that the psychometric properties and task parameters were not identical across all tasks in this paper. Stability and internal consistency were only known for the conditional nonspatial task, and only internal consistency was known for the distributional nonspatial task. Stability and consistency were not known for either spatial task. There were also differences in task parameters such as stimulus presentation times and interstimulus intervals. These differences occurred as we preferred to use tasks that had previously been used in the literature (wherever possible) that had shown reliable assessment of statistical learning.

Nevertheless, this did not appear to have a substantial impact on the observed correlations - for example, the two distributional tasks shared many task parameters but did not correlate more strongly with one another compared with other tasks. However, an ideal experimental design would use tasks with similar and known psychometric properties and would equate more task parameters to better estimate the exact portions of shared and unshared variance. Future research should investigate the psychometric properties of existing and novel measures to ensure optimal measurement of statistical learning across all of its dimensions. It should also further examine the association between different statistical learning tasks with similar parameters. 

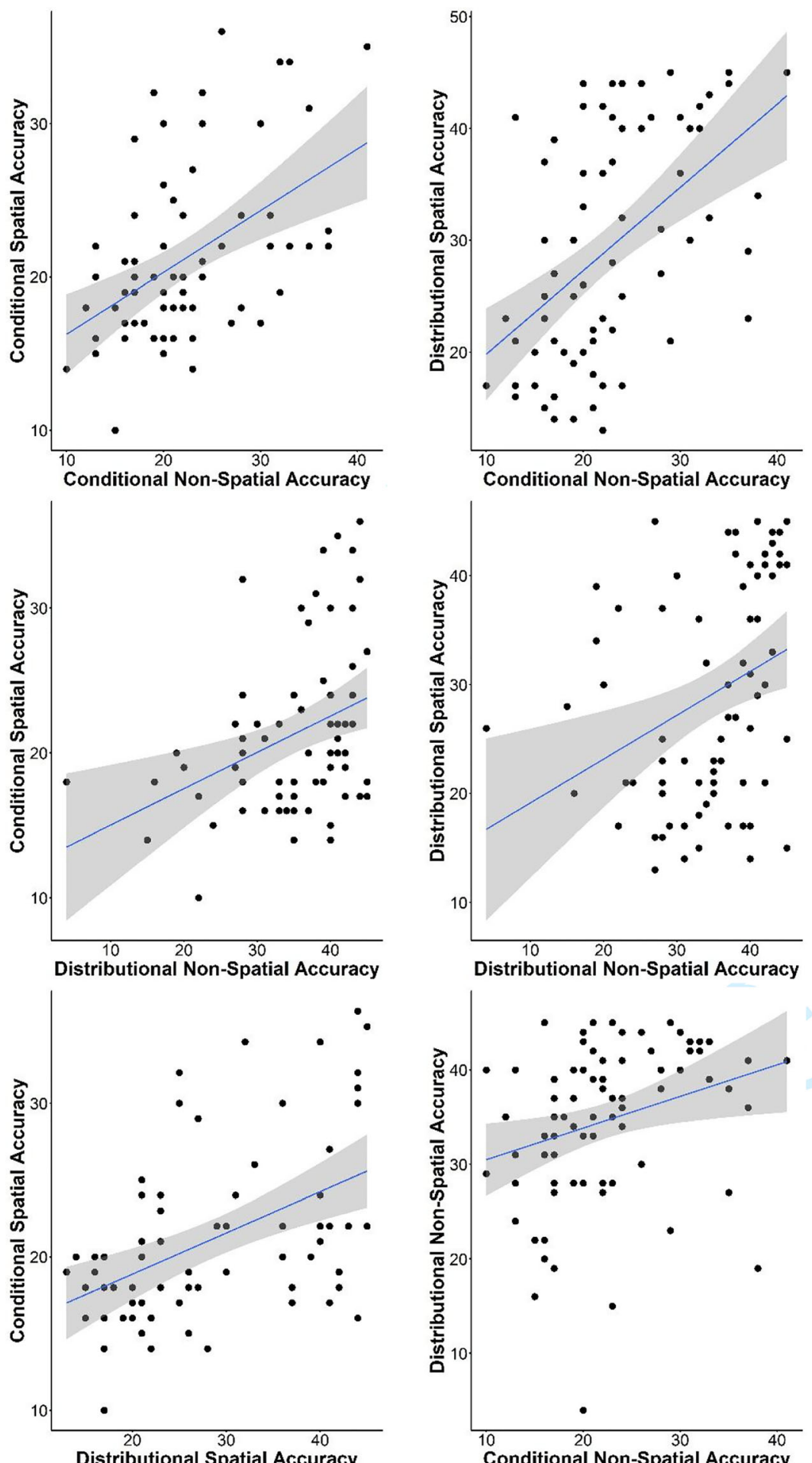

Fig. 4 Correlations between all tasks: Conditional nonspatial and conditional/distributional spatial learning (upper panels), distributional nonspatial and conditional/distributional spatial learning (middle panels), and spatial and nonspatial conditional/distributional learning (lower panels)

It is also worth noting that some of the shared variance across tasks may be due to individual differences in encoding or memory processes rather than statistical learning. Although each task used different shapes to help control for this, it is possible that some individuals may better learn statistical regularities in the visual domain, as they are better at encoding or recalling sensory information. It is possible that statistical learning is inextricably linked with other sensory and memory processes (see Frost et al., 2015) - and may indeed be why higher correlations in statistical learning tasks are seen within 
Table 3. Results of principle component analysis (PCA): Loadings matrix and percentage of variance explained

\begin{tabular}{lllll}
\hline & Component 1 & Component 2 & Component 3 & Component 4 \\
\hline Conditional nonspatial & 0.52 & -0.44 & 0.32 & 0.66 \\
Conditional spatial & 0.43 & 0.87 & 0.15 & 0.17 \\
Distributional nonspatial & 0.51 & -0.09 & -0.85 & -0.05 \\
Distributional spatial & 0.54 & -0.18 & 0.39 & -0.73 \\
Variance explained (\%) & $56.8 \%$ & $18.3 \%$ & $13.6 \%$ & $11.4 \%$ \\
\hline
\end{tabular}

modality (rather than between). Future research should continue to investigate within-modality statistical learning using online measures to better disentangle the relationship between memory and statistical learning (Siegelman, Bogaerts, Kronenfeld, \& Frost, 2018).

\section{Conclusion}

In this paper, we provided the first evidence of a relationship between the ability to extract different forms of visual statistical regularities. We demonstrated that the ability to learn conditional and distributional regularities across time and space is part of one unified visual statistical learning ability, but is also subject to domain-specific constraints reflecting individual skills in learning different types of regularities.

\section{References}

Chen, L., \& Vroomen, J. (2013). Intersensory binding across space and time: A tutorial review. Attention, Perception, \& Psychophysics, 75(5), 790-811. doi:https://doi.org/10.3758/s13414-013-0475-4

Conway, C. M., \& Christiansen, M. H. (2005). Modality-constrained statistical learning of tactile, visual, and auditory sequences. Journal of Experimental Psychology: Learning, Memory, and Cognition, 31(1), 24-39.

Conway, C. M., \& Christiansen, M. H. (2006). Statistical learning within and between modalities: Pitting abstract against stimulus-specific representations. Psychological Science, 17(10), 905-912.

Fiser, J., \& Aslin, R. N. (2001). Unsupervised statistical learning of higher-order spatial structures from visual scenes. Psychological Science, 12(6), 499-504. doi:https://doi.org/10.1111/1467-9280. 00392

Frost, R., Armstrong, B. C., \& Christiansen, M. H. (2019). Statistical learning research: A critical review and possible new directions. Psychological Bulletin, 145(12), 1128-1153. doi: https://doi.org/ 10.1037/bul0000210

Frost, R., Armstrong, B. C., Siegelman, N., \& Christiansen, M. H. (2015). Domain generality versus modality specificity: The paradox of statistical learning. Trends in Cognitive Sciences, 19(3), 117-125.

Growns, B., \& Martire, K. A. (in press). Forensic feature-comparison expertise: Statistical learning facilitates visual comparison performance. Journal of Experimental Psychology: Applied, 1-38. https://psyarxiv.com/pzfjb/

Mitchel, A. D., \& Weiss, D. J. (2011). Learning across senses: Crossmodal effects in multisensory statistical learning. Journal of
Experimental Psychology: Learning, Memory \& Cognition, 37(5), 1081.

Mollon, J. D., Bosten, J. M., Peterzell, D. H., \& Webster, M. A. (2017). Individual differences in visual science: What can be learned and what is good experimental practice? Vision Research, 141, 4-15. doi:https://doi.org/10.1016/j.visres.2017.11.001

Newport, E. L., \& Aslin, R. N. (2004). Learning at a distance I. Statistical learning of non-adjacent dependencies. Cognitive Psychology, 48(2), 127-162.

Pavlidou, E., \& Bogaerts, L. (2019). Implicit statistical learning across modalities and its relationship with reading in childhood. Frontiers in Psychology, 10, 1834.

Perfors, A., \& Kidd, E. (2018). What drives individual differences in statistical learning? The role of perceptual fluency and familiarity. PsyArXiv. doi:https://doi.org/10.31234/osf.io/7jvx8

Raviv, L., \& Arnon, I. (2018). The developmental trajectory of children's auditory and visual statistical learning abilities: modality-based differences in the effect of age. Journal of Developmental Science, 21(4), e12593.

Recanzone, G. H. (2009). Interactions of auditory and visual stimuli in space and time. Hearing Research, 258(1/2), 89-99.

Romberg, A. R., \& Saffran, J. R. (2013). All together now: Concurrent learning of multiple structures in an artificial language. Cognitive Science, 37(7), 1290-1320.

Siegelman, N., Bogaerts, L., Christiansen, M. H., \& Frost, R. (2017a). Towards a theory of individual differences in statistical learning. Philosophical Transactions of the Royal Society B: Biological Sciences, 372, 1-10.

Siegelman, N., Bogaerts, L., \& Frost, R. (2017b). Measuring individual differences in statistical learning: Current pitfalls and possible solutions. Behavior Research Methods, 49(2), 418-432.

Siegelman, N., Bogaerts, L., Kronenfeld, O., \& Frost, R. (2018). Redefining "learning" in statistical learning: What does an online measure reveal about the assimilation of visual regularities? Cognitive Science, 42, 692-727.

Siegelman, N., \& Frost, R. (2015). Statistical learning as an individual ability: Theoretical perspectives and empirical evidence. Journal of Memory and Language, 81, 105-120.

Thiessen, E. D., \& Erickson, L. C. (2013). Beyond word segmentation: A two-process account of statistical learning. Journal of Current Directions in Psychological Science, 22(3), 239-243.

Thiessen, E. D., Kronstein, A. T., \& Hufnagle, D. G. (2013). The extraction and integration framework: A two-process account of statistical learning. Psychological Bulletin, 139(4), 792-814.

Wetzels, R., Matzke, D., Lee, M. D., Rouder, J. N., Iverson, G. J., \& Wagenmakers, E.-J. (2011). Statistical evidence in experimental psychology: An empirical comparison using $855 t$ tests. Journal of Perspectives on Psychological Science, 6(3), 291-298.

Publisher's note Springer Nature remains neutral with regard to jurisdictional claims in published maps and institutional affiliations. 